\title{
Temporary External Fixation for Forearm Fractures in Damage Control Orthopedics Procedures: Use of a Simplified Frame
}

\author{
Laurent Mathieu*, Antoine Grosset, Ismaël Hassan Farah, Nicolas de l'Escalopier, \\ James-Charles Murison and Sylvain Rigal
}

Department of Orthopaedic, Traumatology and Reconstructive Surgery, Percy Military Hospital, France

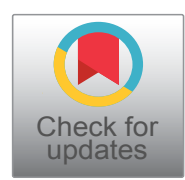

*Corresponding author: Laurent Mathieu, Department of Orthopaedic, Traumatology and Reconstructive Surgery, Percy Military Hospital, 101 Avenue Henri Barbusse 92140 Clamart, France, Tel: 00-33-1-4146-6157, E-mail: laurent_tom2@yahoo.fr

\begin{abstract}
External fixation of the forearm may be indicated for Gustilo grade $3 \mathrm{~B}-\mathrm{C}$ open fractures or in unstable polytraumatized patients as part of a damage-control orthopedics (DCO) strategy to prepare secondary conversion to internal fixation by plates. Because conventional forearm frames with separate stabilization of the radius and ulna do not meet DCO requirements, the authors propose to use a single unilateral frame. A case of severe forearm open fracture related to a gunshot wound is presented to illustrate the advantages of this simplified frame.
\end{abstract}

\section{Keywords}

Damage control orthopedics, Forearm fractures, Temporary external fixation, Secondary internal fixation

\section{Introduction}

Open reduction and internal fixation by plates is the surgical treatment of choice for adult forearm diaphyseal fractures. However, EF may be required for severe open fractures or in unstable polytraumatized patients for whom prolonged operating time is detrimental $[1,2]$. The problems of using EF in the forearm include pintrack infection or radio-ulnar synostosis, nerve damage due to insufficient anatomical exposure, and a relatively high rate of nonunion [1-3]. Thus, immediate forearm $\mathrm{EF}$ and planned conversion to internal fixation is often used in sequential damage control orthopedics (DCO) strategies [4-6]. Because conventional forearm frames with separate stabilization of the radius and ulna do not meet DCO requirements, the authors propose to use a single unilateral frame derived from the one-bone fore- arm concept. The aim is to provide adequate longitudinal and rotational stability to the forearm, while limiting morbidity related to nerve damage and to pin-track infection or fracture.

\section{Surgical Technique}

The forearm unilateral frame is composed of two screws inserted in the proximal ulna and two screws inserted in the distal radius connected by a single dorsal carbon rod/tube (Figure 1). Screws must be placed distant from the fracture site to reduce the risk of deep infection and permit early conversion to internal fixation [4-6]. Since both radius and ulna are small diameter bones, 3.0 diameter screws should be preferred to minimize the possible danger of iatrogenic fracture. However, 4.0 diameter screws can be safely implanted in metaphyseal areas such as proximal ulna and distal radius.

The surgeon must pronate the forearm to apply the fixator dorsally. To be easily connected to radial screws, ulnar screws are implanted laterally or dorsolaterally in the proximal third of the ulna. They can be inserted percutaneously through the dorsal third of the extensor carpi ulnaris muscle, or the muscle can be divided by a limited open approach. At this level, careful drilling and screw insertion is crucial to avoid secondary radio-ulnar synostosis [3]. Conversely, open screw insertion is mandatory on the radius to avoid tendon or nerve damage. Screws are inserted on the dorsolateral side of the radius, just proximal to the Lister's tubercle in a muscle- and tendon-free triangle between extensor carpi 

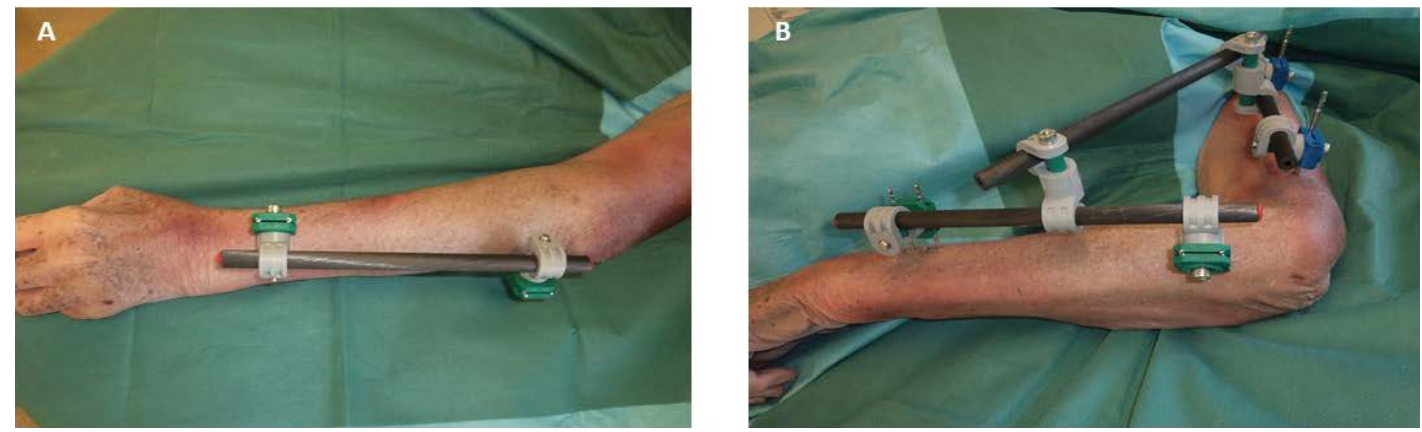

Figure 1: Temporary unilateral radioulnar frame. A) Dorsal view; B) Lateral view with associated spanning elbow fixation.
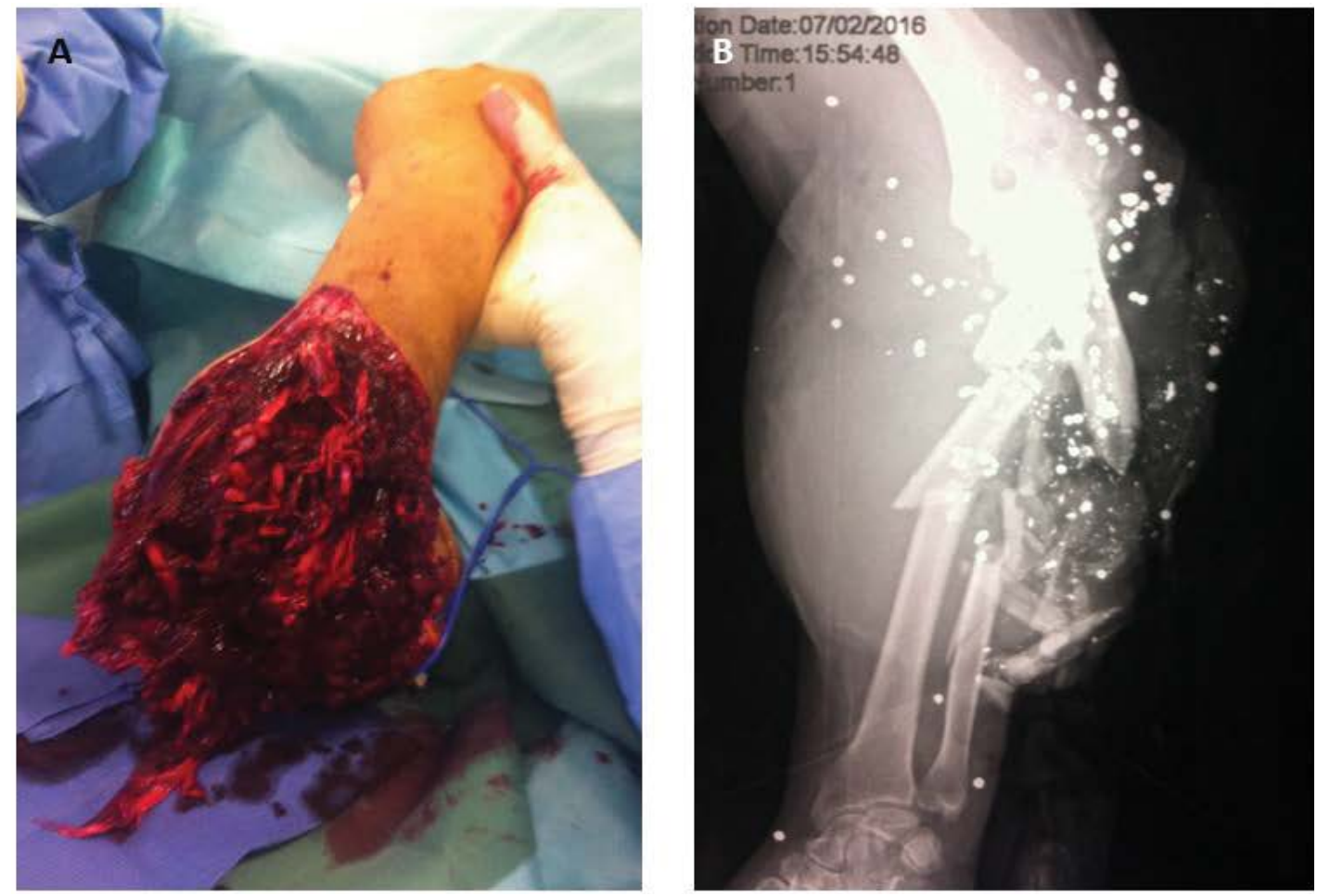

Figure 2: Gunshot wound of the left forearm. A) Preoperative radiographs showing highly comminuted fracture of the mid ulna and bifocal fracture of the proximal radius; B) Clinical aspect before débridement and external fixation.

radialis brevis and extensor pollicis longus tendons [7]. This distal screw implantation is easier and safer for the radial nerve compare to proximal or midshaft implantations. Open clamps and carbon rod should be positioned close to the skin to achieve sufficient stability [4]. After frame application, approximative reduction is achieved by longitudinal traction under fluoroscopy. The forearm is placed in neutral position of supination-pronation or slight pronation. Once reduction is completed clamps are closed and locked.

For patients with multi-focal upper extremity injuries, this modular unilateral frame can be easily connected to a humeral lateral frame for spanning fixation of the elbow, or to dorsolateral screws implanted in the second metacarpal bone for spanning fixation of the wrist (Figure 1).

\section{Case Study}

A 65-year-old, right-hand dominant, man was admitted following a gunshot wound that occurred after a hunting accident. He sustained a Gustilo grade 3B open fracture of his left forearm with an extended dorsomedial wound [8]. Muscles of the dorsal compartment were severely injured and the wound highly contaminated. A loss of fingers extension and fifth finger numbness were noticed, but the radial pulse was present. Radiographs showed a comminuted fracture of the mid ulna with bone loss associated to a complex bifocal fracture of the proximal radius (Figure 2).

Surgical exploration revealed partial transection of the extensor carpi radialis brevis, extensive loss of extensor communis digitorum, extensor digiti minimi and extensor carpi ulnaris with contusion of the ulnar nerve. The supinator muscle was dilacerated with rupture of the posterior interosseous nerve. After meticulous débridement and copious irrigation with normal saline, the patient underwent temporary EF of the forearm using a dorsal radioulnar frame connected to a spanning fixation of the wrist. Direct closure was impossible due to a large dorsal skin defect (Figure 3). 

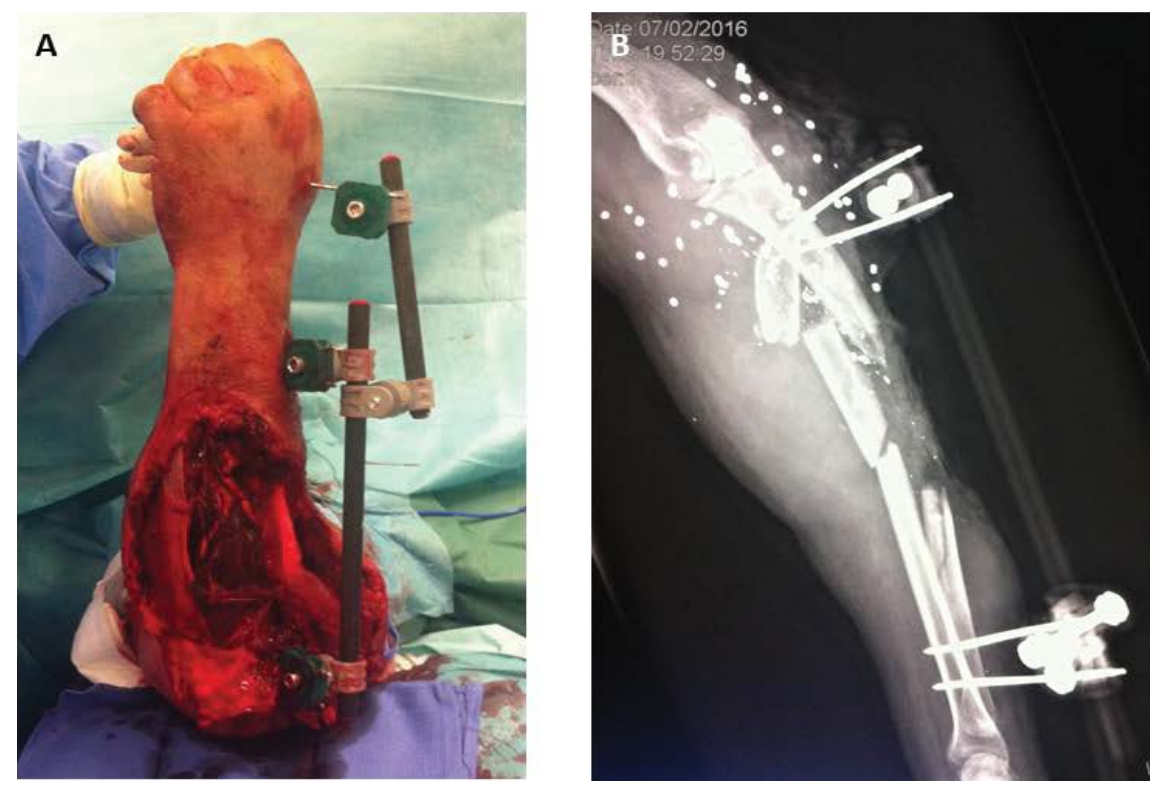

Figure 3: Initial DCO procedure. A) Extensive soft-tissue defect after débridement and unilateral radioulnar frame with spanning fixation of the wrist; B) Postoperative film showing approximative reduction and large segmental defect on the ulna.
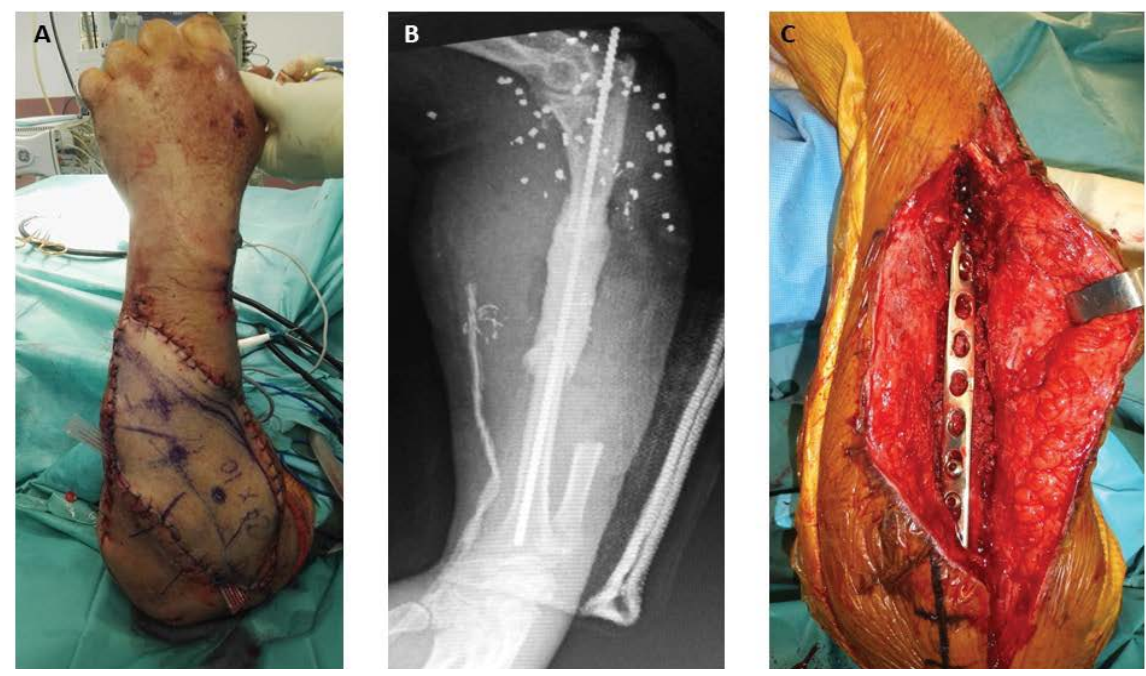

Figure 4: Two-stages reconstruction. A) Vascularized flap transfer; B) First stage of the one bone forearm procedure; C) Cancellous bone grafting and definitive internal fixation.

Considering the midshaft defect on the ulna and the severity of the proximal radius fracture a one bone forearm procedure with staged reconstruction was decided. One-step reconstruction using a composite fibular free flap was rejected because an early polybacterial infection occurred. Two subsequent débridements were needed before soft-tissue coverage could be achieved by a vascularized anterolateral tight flap. In the same procedure, the external fixator was removed and the proximal ulna was connected to the distal radius using an intramedullary Kirschner nail combined to a cement spacer applied in the remaining bone defect to maintain forearm length (Figure 4). Soft-tissue management was facilitated by the unilateral frame without loss reduction during repeated débridement procedures. Oral antibiotic treatment was prescribed during 6 weeks before definitive internal fixation and cancellous iliac bone grafting were performed according to the Masquelet induced membrane technique [9].
At the last follow-up of 8 months, cosmetic aspect was excellent and bone union was achieved (Figure 5). The patient had full range of motion of the elbow and wrist, but presented a limited extension of the fingers. He was very satisfied of the functional outcome and declined tendon transfer surgery.

\section{Discussion}

Indications for temporary forearm EF mainly include open fractures with considerable soft tissue damage, Gustilo grade $3 \mathrm{~B}$ or $3 \mathrm{C}$, or fractures complicated by acute compartment syndrome after fasciotomies have been completed [8]. In both cases, direct primary closure cannot be carried out which precludes early plate fixation [1]. Temporary EF should also be considered for closed fractures in three situations requiring application of the DCO concept: Unstable polytraumatized patients for whom prolonged operating time associated with immediate open reduction and internal fixation may be 

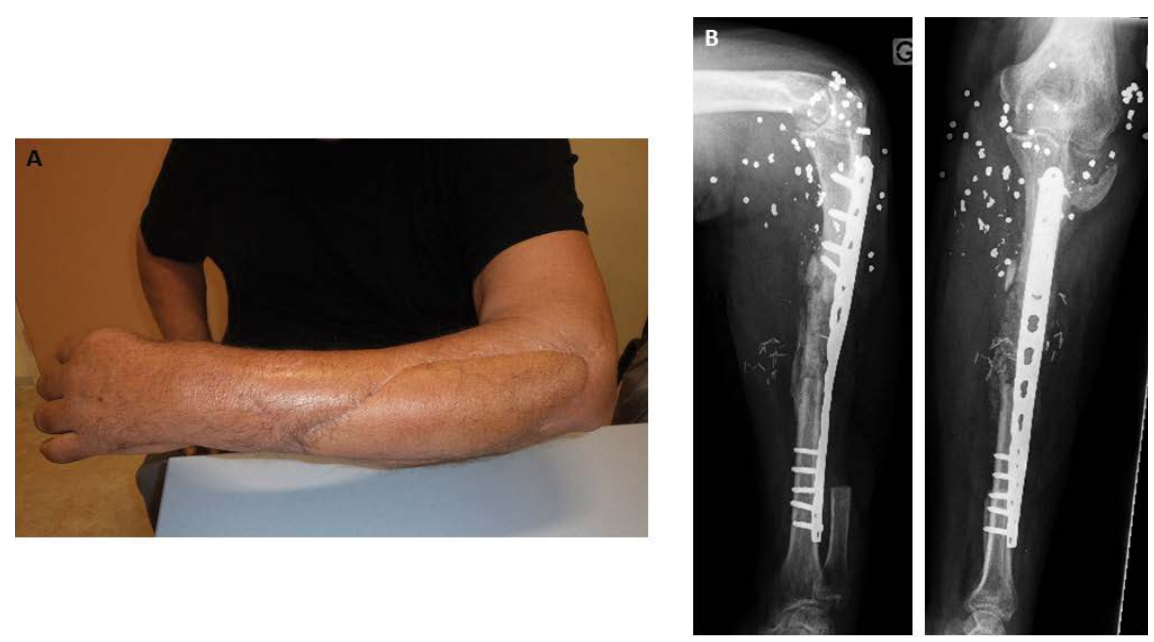

Figure 5: A) Aesthetic appearance after 8 months; B) Radiographs showing bone union at the last follow-up.

detrimental; highly comminuted fractures or multi-focal injuries of the upper extremity that should be managed by an experienced trauma surgeon; and limited resources with lack of internal fixation means, as encountered in austere environments or massive casualties [4]. However, splinting of closed fractures may be a valid alternative in these situations [5].

According to the DCO principles, temporary stabilization of long bones should be achieved by unilateral frames with only two screws on the two main fragments $[4,6]$. At the forearm level, stabilization of both radius and ulna usually require two individual devices which can be united to prevent rotational range of motion. Because screw implantation in the mid and proximal radius is delicate and at risk for the posterior interosseous nerve, temporary EF may be limited to the ulna fracture - especially when associated to elbow spanning fixation $[7,10]$. However, rotational instability due to the $a b-$ sence of radius stabilization may cause pain and soft tissue lesion during patient mobilization. To optimize temporary forearm $\mathrm{EF}$, the authors proposed to use a single dorsolateral frame implanted on the proximal ulna and the distal radius. This simplified technique is derived from salvage one-bone forearm procedures dedicated to treating long segmental bone defects resulting from trauma, infection or tumor resection $[11,12]$. The aim is to provide adequate longitudinal and rotational stability to the forearm, while limiting morbidity related to nerve damage and to pin-track infection or fracture.

Complications after temporary EF include mainly loss of reduction between initial and revision surgery and pin-track infection. Loss of reduction may be due to insufficient frame stiffness which can be augmented by reduction of the bone to rod distance. However, in a DCO procedure the aim of EF is not to achieve anatomical reduction and bone union, but to maintain longitudinal and rotational stability for few days until definitive fixation is performed. The risk of pin-track infection is reduced with the use of a 4 screws frame compared to classic forearm frames requiring a minimum of 8 screws.
Furthermore, iatrogenic nerve injuries or pin-track fractures should be limited with the screw placement described here.

When temporary forearm EF is decided upon as initial treatment, the date for conversion to definitive fixation should be planned as quickly as possible. Indeed, it is recommended that internal fixation by plates should be performed within 15 days, once the soft-tissue envelope is suitable and there are no signs of wound or pin tract infection. In case of severe open fractures, the soft tissue injury must have resolved before internal fixation can be ruled out. Repeated marginal débridement is often required until the wound becomes clean enough to perform skin coverage by direct suture, grafting or flap transfer, ideally before the $7^{\text {th }}$ day post-trauma [4-6]. Despite the unilateral frame was converted to a definitive one-bone-forearm procedure in the case presented, a conventional internal fixation of both bones could be performed in less dramatic clinical presentations when forearm rotation can be restored.

\section{Conclusion}

Damage control orthopedics is a concept of fluid thinking, thinking outside the box and temporizing fractures for optimal care after soft tissues are managed. For temporary forearm EF, the authors propose to use a single dorsal radioulnar frame. This quick, easy and effective technique limits the risk of complications and facilitates early conversion to internal fixation by plates.

\section{Conflict of Interest}

The authors declare no conflict of interest in relation with this paper.

\section{Funding}

There is no funding source.

\section{Ethical Approval}

This article does not contain any studies with human participants or animals performed by any of the authors. 


\section{References}

1. Helber MU, Ulrich C (2000) External fixation in forearm shaft fractures. Injury 30: 45-47.

2. Schuind F, Andrianne Y, Burny F (1991) Treatment of forearm fractures by Hoffmann external fixation. A study of 93 patients. Clin Orthop Relat Res 197-204.

3. Kanakaris N, Tsoutseos N (2007) Proximal radio-ulnar synostosis at the pin-track site after external fixation of the forearm. Eur J Trauma Emerg Surg 33: 293-296.

4. Mathieu L, Bazile F, Barthélémy R, Duhamel P, Rigal S (2011) Damage control orthopaedics in the context of battlefield injuries: The use of temporary external fixation on combat trauma soldiers. Orthop Traumatol Surg Res 97: 852-859.

5. Mathieu L, Bertani A, Gaillard C, Chaudiera P, Ollat D, et al. (2014) Combat-related upper extremity injuries: Surgical management specificities on the theatres of operations. Chir Main 33: 174-182.

6. Mathieu L, Ouattara N, Poichotte A, Saint-Macari E, Barbier O, et al. (2014) Temporary and definitive external fixation of war injuries: Use of a French dedicated fixator. Int Orthop 38: 1569-1576.

7. Gausepohl T, Koebke J, Pennig D, Hobrecker S, Mader K (2000) The anatomical base of unilateral external fixation in the upper limb. Injury 31: 11-20.

8. Gustilo RB, Mendoza RM, Williams DN (1984) Problems in the management of type III (severe) open fractures: A new classification of type III open fractures. J Trauma 24: 742-746.

9. Masquelet AC, Begue T (2010) The concept of induced membrane for reconstruction of long bone defects. Orthop Clin North Am 41: 27-37.

10. Kulesh PN, Fletcher MDA, Solomin LN (2015) Avoidance of external fixation pin reduced rotational stiffness in the forearm; a cadaver study of soft tissue displacement relative to the varying position of radius and ulna fixation. SICOT J 1: 3.

11. Reid RL, Baker GI (1973) The single-bone forearm - a reconstructive technique. Hand 5: 214-219.

12. Castle ME (1974) One-bone forearm. J Bone Joint Surg Am 56: $1223-1227$. 